\title{
UNCERTAIN SYSTEMS, BEHAVIOURS AND QUADRATIC DIFFERENTIAL FORMS ${ }^{1}$
}

\author{
Ian R. Petersen* Jan C. Willems ** \\ * School of Electrical Engineering, Australian Defence Force Academy, \\ Campbell, 2600, Australia, Phone +61 2 62688446, FAX +61 2 \\ 62688443,email:irp@ee.adfa.oz.au \\ ** Research Institute for Mathematics and Computing Science, P.O. Box \\ 800, 9700 AV Groningen, The Netherlands
}

\begin{abstract}
This paper considers uncertain systems from a behavioural point of view defined via quadratic differential forms. This uncertainty definition is closely related to the integral quadratic constraint uncertainty description commonly found in robust control theory. The paper presents a frequency domain condition for the set of behaviours of a given uncertain system to contain the set of behaviours of another given uncertain system. This result is useful in uncertainty modelling problems in which one wishes to consider the trade off between model complexity and model conservatism.
\end{abstract}

Keywords: Uncertain Systems; Behaviours; Quadratic Differential Forms; Equivalent Realizations; Uncertainty Modelling; Differential Inequality Systems.

\section{INTRODUCTION}

If we take a behavioural approach to system modelling, we can regard a system as being characterized by a corresponding set of possible trajectories; e.g., see (Polderman and Willems, 1998). This idea can also be applied to uncertain system models in which the model is required to capture a range of possible process dynamics; e.g, see (D'Andrea and Paganini, 1993; D'Andrea et al., 1993; D'Andrea and Paganini, 1994; Paganini and Doyle, 1994) in which some different approaches to modelling uncertain systems from a behavioural point of view are considered. This paper considers a new class of systems modelled within a behavioural framework which are motivated by the idea of representing uncertainty in system dynamics. In this new class of behavioural systems, the behaviour sets being considered are defined in terms of quadratic differential forms; e.g., see (Willems and Trentelman, 1998). This description can be regarded as a behavioural generalization of the frequency domain integral quadratic constraint (IQC)

1 This work was supported by the Australian Research Council uncertainty description for the case of a single uncertainty constraint; e.g., see (Boyd et al., 1994; Megretski and Rantzer, 1997). We will refer to these systems as Differential Inequality Systems. In particular, this system description allows for dynamics which are nonlinear and time-varying. Also, the system description considered in this paper is closely related to the time-domain IQC uncertainty description; e.g., see (Petersen et al., 2000). The time domain IQC uncertainty description has been found to yield tractable solutions to problems of minimax optimal control and state estimation; e.g., see (Petersen et al., 2000).

The paper is concerned characterizing equivalences and inclusion relations for differential inequality systems described in terms of quadratic differential forms. One differential inequality system is said to be a superset of another if the set of behaviours of the first system contains the set of behaviours of the second system. Also, if two differential inequality systems described in terms of two quadratic differential forms have the same set of behaviours then they are equivalent. In problems of uncertainty modelling in which one begins with a differential inequality sys- 
tem model of a process, one might seek to find a simpler differential inequality system model which is equivalent to the original system model. Also, in some circumstances, one might be prepared to replace the original differential inequality system model with a simpler differential inequality system model which is a superset of the original differential inequality system model.

It should be noted that a very complete theory of minimality and equivalence for a class of uncertain systems has been developed in the papers (Beck, 1994; Beck et al., 1996; Beck and D'Andrea, 1997; Beck and Doyle, 1999). However these papers consider a different class of uncertain systems than the differential inequality systems considered in this paper. Also, our notions of system equivalence and inclusion are quite different than those considered in these papers.

The remainder of the paper proceeds as follows. In Section 2, we introduce the class of differential inequality systems under consideration. We also include definitions concerning the relationship between two differential inequality systems and the equivalence of two differential inequality systems. This section also recalls a standard S-procedure result and a result on quadratic differential forms from (Willems and Trentelman, 1998). These preliminary results will be used in the proof of our main result.

In Section 3, we present our main result characterizing when a given differential inequality system is a superset of another differential inequality system. This result is given in terms of a frequency domain condition. This in turn leads to a frequency domain condition for the equivalence of two differential inequality systems. In Section 4, we present a simple example which illustrates our main results.

\section{DEFINITIONS AND PRELIMINARY RESULTS}

We first introduce our definition of differential inequality system. Such systems can be regarded as a type of uncertain system defined within a behavioural framework; e.g., see (Polderman and Willems, 1998) for further details on the behavioural approach to the modelling of dynamical systems. In this definition, $\mathbb{R}_{S}^{q \times q}[\zeta, \eta]$ denotes the set of real symmetric $q \times q$ polynomial matrices in the (commuting) indeterminates $\zeta$ and $\eta$. An element $\Phi \in \mathbb{R}_{s}^{q \times q}[\zeta, \eta]$ is thus given by

$$
\Phi(\zeta, \eta)=\sum_{k, \ell} \phi_{k \ell} \zeta^{k} \eta^{\ell}
$$

where

$$
\phi_{k, \ell}=\phi_{\ell, k}^{T}
$$

Definition 1. Let $\Phi \in \mathbb{R}_{S}^{q \times q}[\zeta, \eta]$ be given. The differential inequality system $\Sigma_{\Phi}$ is defined by $\Sigma_{\Phi}:=$ $\left(\mathbb{R}, \mathbb{R}^{q}, \mathfrak{B}_{\Phi}\right)$ with

$$
\mathfrak{B}_{\Phi}:=\left\{w \in \mathfrak{D}\left(\mathbb{R}, \mathbb{R}^{q}\right): \int_{-\infty}^{\infty} Q_{\Phi}(w) d t \geq 0\right\} .
$$

Here

$$
\begin{aligned}
\mathfrak{D}\left(\mathbb{R}, \mathbb{R}^{q}\right) & :=\left\{\begin{array}{l}
w \in \mathfrak{C}^{\infty}\left(\mathbb{R}, \mathbb{R}^{q}\right): \\
w \text { has compact support }
\end{array}\right\}, \\
Q_{\Phi}(w) & :=\sum_{k, \ell}\left(\frac{d^{k} w}{d t^{k}}\right)^{T} \phi_{k \ell}\left(\frac{d^{\ell} w}{d t^{\ell}}\right) .
\end{aligned}
$$

Note that $\Sigma_{\Phi}$ is parametrized by $\Phi$.

Remarks To illustrate the above definition, consider a linear system described by an equation of the form $R\left(\frac{d}{d t}\right) w=0$; e.g., see (Polderman and Willems, $1998)$. Here $R(s)$ is a real polynomial matrix. Now observe that

$$
\begin{aligned}
& \left\{w \in \mathfrak{D}\left(\mathbb{R}, \mathbb{R}^{q}\right): R\left(\frac{d}{d t}\right) w=0\right\} \\
& =\left\{\begin{array}{c}
w \in \mathfrak{D}\left(\mathbb{R}, \mathbb{R}^{q}\right): \\
\left.\int_{-\infty}^{\infty}-\left(R\left(\frac{d}{d t}\right) w\right)^{T} R\left(\frac{d}{d t}\right) w d t \geq 0\right\}
\end{array}\right. \\
& =\left\{w \in \mathfrak{D}\left(\mathbb{R}, \mathbb{R}^{q}\right): \int_{-\infty}^{\infty} Q_{\Phi_{R}}(w) d t \geq 0\right\}
\end{aligned}
$$

where $\Phi_{R}(\zeta, \eta)=-R^{T}(\zeta) R(\eta)$. Thus, our class of differential inequality systems includes linear behavioural systems; e.g., see (Polderman and Willems, 1998).

To further motivate the above definition and to relate it to more standard notions of an uncertain system, consider the following simple example. Suppose $u$ is the system input and $y$ is the system output and let

$$
w=\left[\begin{array}{l}
u \\
y
\end{array}\right] \text {. }
$$

Also, let

$$
\Phi(\zeta, \eta)=\left[\begin{array}{ll}
0 & 1 \\
1 & 0
\end{array}\right]
$$

Then the corresponding differential inequality system is characterized by the set of behaviours

$$
\left\{[u, y] \in \mathfrak{D}\left(\mathbb{R}, \mathbb{R}^{q}\right): \int_{-\infty}^{\infty} 2 u y d t \geq 0\right\} .
$$

This system includes all sector bounded static nonlinearities of the form $y=f(u)$ where $\sigma f(\sigma) \geq 0$ for all $\sigma$.

Our main aim is to look at conditions under which a given differential inequality system is a superset of another given differential inequality system in the following sense.

Definition 2. Suppose $\Sigma_{\Phi_{1}}$ and $\Sigma_{\Phi_{2}}$ are two differential inequality systems defined as in Definition 1. Then, we write $\Sigma_{\Phi_{1}} \leq \Sigma_{\Phi_{2}}$ if $\mathfrak{B}_{\Phi_{1}} \subset \mathfrak{B}_{\Phi_{2}}$. Also, we write $\Sigma_{\Phi_{1}}=\Sigma_{\Phi_{2}}$ if $\mathfrak{B}_{\Phi_{1}}=\mathfrak{B}_{\Phi_{2}}$. Clearly, $\Sigma_{\Phi_{1}}=$ $\Sigma_{\Phi_{2}}$ if and only if $\Sigma_{\Phi_{1}} \leq \Sigma_{\Phi_{2}}$ and $\Sigma_{\Phi_{2}} \leq \Sigma_{\Phi_{1}}$ Note 
that this definition defines an equivalence relation on $\mathbb{R}_{s}^{q \times q}[\zeta, \eta]$. Indeed, if $\Sigma_{\Phi_{1}}=\Sigma_{\Phi_{2}}$, we write $\Phi_{1} \sim \Phi_{2}$.

Given two differential inequality systems $\Sigma_{\Phi_{1}}$ and $\Sigma_{\Phi_{2}}$, our main result is concerned with the questions: When is $\Sigma_{\Phi_{1}} \leq \Sigma_{\Phi_{2}}$ and when is $\Sigma_{\Phi_{1}}=\Sigma_{\Phi_{2}}$. This result involves the use of the following well known Sprocedure theorem for two quadratic forms (e.g., see (Yakubovich, 1973; Petersen et al., 2000) for proof).

Lemma 3. Let $\mathscr{X}$ be a real linear vector space and $\mathscr{G}_{1}(x), \mathscr{G}_{2}(x)$ be quadratic functionals on $\mathscr{X}$. That is, $\mathscr{G}_{1}(x)$ and $\mathscr{G}_{2}(x)$ are functionals of the form

$$
\begin{aligned}
& \mathscr{G}_{1}(x)=G_{1}(x, x)+g_{1}(x)+\gamma_{1}, \\
& \mathscr{G}_{2}(x)=G_{2}(x, x)+g_{2}(x)+\gamma_{2}
\end{aligned}
$$

where $G_{1}\left(x_{1}, x_{2}\right)$ and $G_{2}\left(x_{1}, x_{2}\right)$ are bilinear forms on $\mathscr{X} \times \mathscr{X}, g_{1}(x), g_{2}(x)$ are linear functionals on $\mathscr{X}$, and $\gamma_{1}, \gamma_{2}$ are constants. Assume that there exists a vector $x_{0} \in \mathscr{X}$ such that $\mathscr{G}_{1}\left(x_{0}\right)>0$. Then, the following conditions are equivalent:

(i) $\mathscr{G}_{2}(x) \geq 0$ for all $x$ such that $\mathscr{G}_{1}(x) \geq 0$;

(ii) There exists a constant $\tau \geq 0$ such that

$$
\mathscr{G}_{2}(x)-\tau \mathscr{G}_{1}(x) \geq 0
$$

for all $x \in \mathscr{X}$.

Notation Associated with a quadratic differential form $Q_{\Phi}(w)$ defined as in (1) is a corresponding polynomial matrix $\partial \Phi \in \mathbb{R}_{s}^{q \times q}[\xi]$ defined by

$$
\partial \Phi(\xi):=\Phi(-\xi, \xi)
$$

Lemma 4. Consider a quadratic differential form $Q_{\Phi}(w)$ defined as above. Then

$$
\int_{-\infty}^{\infty} Q_{\Phi}(w) d t \geq 0 \quad \forall w \in \mathfrak{D}\left(\mathbb{R}, \mathbb{R}^{q}\right) .
$$

if and only if $\partial \Phi(i \omega) \geq 0 \quad \forall \omega \in \mathbb{R}$,

PROOF. This result follows directly from Proposition 5.2 of (Willems and Trentelman, 1998).

\section{THE MAIN RESULT}

We now present our main result.

Theorem 5. Let $\Phi_{1}, \Phi_{2} \in \mathbb{R}_{S}^{q \times q}[\zeta, \eta]$ be given. Then $\Sigma_{\Phi_{1}} \leq \Sigma_{\Phi_{2}}$ if and only if either of the following conditions hold:

(i) There exists a $\tau \geq 0$ such that

$$
\tau \partial \Phi_{1}(i \omega) \leq \partial \Phi_{2}(i \omega) \quad \forall \omega \in \mathbb{R} .
$$

(ii)

$$
\partial \Phi_{1}(i \omega) \leq 0 \quad \forall \omega \in \mathbb{R}
$$

and

$$
m(-i \omega)^{T} \partial \Phi_{2}(i \omega) m(i \omega) \geq 0
$$

for all $m(\xi) \in \mathbb{R}^{q}[\xi]$ such that $\partial \Phi_{1}(\xi) m(\xi)=0$.

The proof of this theorem relies on the following lemmas.

Lemma 6. Let $\Phi \in \mathbb{R}_{s}^{q \times q}[\zeta, \eta]$ be given. Then there exists a $w \in \mathfrak{D}\left(\mathbb{R}, \mathbb{R}^{q}\right)$ such that

$$
\int_{-\infty}^{\infty} Q_{\Phi}(w) d t>0
$$

if and only if there exists an $\omega \in \mathbb{R}$ such that $\partial \Phi(i \omega) \not \leq$ 0 .

PROOF. This proof follows the proof of Theorem 3.1 and Proposition 5.2 in (Willems and Trentelman, 1998). Suppose $\partial \Phi\left(i \omega_{0}\right) \not \leq 0$. Then there exists an $a \in \mathbb{C}^{q}$ such that

$$
a^{*} \partial \Phi\left(i \omega_{0}\right) a>0 .
$$

Now define a sequence of functions $w_{N}(t) \in \mathfrak{D}\left(\mathbb{R}, \mathbb{C}^{q}\right)$, $N=1,2, \ldots$, by

$$
w_{N}(t)= \begin{cases}e^{i \omega_{0} t} a & \text { for }|t| \leq \frac{2 \pi N}{\omega_{0}}, \\ \tilde{w}\left(t+\frac{2 \pi N}{\omega_{0}}\right) & \text { for } t<-\frac{2 \pi N}{\omega_{0}}, \\ \tilde{w}\left(t-\frac{2 \pi N}{\omega_{0}}\right) & \text { for } t>\frac{2 \pi N}{\omega_{0}} .\end{cases}
$$

Here $\tilde{w}(\cdot)$ is a function chosen independently of $N$ so that $w_{N}(t) \in \mathfrak{D}\left(\mathbb{R}, \mathbb{C}^{q}\right)$ for all $N$. This is possible because of the periodic nature of $w_{N}(t)$ for $|t| \leq \frac{2 \pi N}{\omega_{0}}$. Then

$$
\int_{-\infty}^{\infty} Q_{\Phi}\left(w_{N}\right) d t=\frac{4 \pi N}{\omega_{0}} a^{*} \partial \Phi\left(i \omega_{0}\right) a+E
$$

with $E$ independent of N. Hence, $a^{*} \partial \Phi\left(i \omega_{0}\right) a>0$, implies that $\int_{-\infty}^{\infty} Q_{\Phi}\left(w_{N}\right) d t>0$ for sufficiently large $N$. A similar conclusion can be obtained using a real signal $w(t)$ by taking real and imaginary parts of $w_{N}$. Thus, we can conclude that there exists a $w \in$ $\mathfrak{D}\left(\mathbb{R}, \mathbb{R}^{q}\right)$ such that (6) holds.

Conversely, suppose there exists a $w \in \mathfrak{D}\left(\mathbb{R}, \mathbb{R}^{q}\right)$ such that (6) holds. Let $\hat{w}(i \omega)$ be the Fourier Transform of $w(t)$. Then using Parseval's Theorem, (6) implies

$$
\int_{-\infty}^{\infty} \hat{w}(-i \omega)^{T} \Phi(-i \omega, i \omega) \hat{w}(i \omega) d \omega>0
$$

Hence, there exists a $\omega_{0} \in \mathbb{R}$ such that

$$
\hat{w}\left(-i \omega_{0}\right)^{T} \partial \Phi\left(i \omega_{0}\right) \hat{w}\left(i \omega_{0}\right) .
$$

Thus, $\partial \Phi\left(i \omega_{0}\right) \not \leq 0$. This completes the proof of the Lemma. 
Lemma 7. Let $R \in \mathbb{R}^{p \times q}[\xi]$ and $\Phi \in \mathbb{R}_{s}^{q \times q}[\zeta, \eta]$ be given. Then

$$
\begin{aligned}
& \left\{w \in \mathfrak{D}\left(\mathbb{R}, \mathbb{R}^{q}\right): R\left(\frac{d}{d t}\right) w=0\right\} \\
& \subset\left\{w \in \mathfrak{D}\left(\mathbb{R}, \mathbb{R}^{q}\right): \int_{-\infty}^{\infty} Q_{\Phi}(w) d t \geq 0\right\}
\end{aligned}
$$

if and only if

$$
m(-i \omega)^{T} \partial \Phi(i \omega) m(i \omega) \geq 0 \quad \forall \omega \in \mathbb{R}
$$

for all $m(\xi) \in \mathbb{R}^{q}[\xi]$ such that $R(\xi) m(\xi)=0$.

PROOF. Suppose the condition (8) holds and let $w=$ $M\left(\frac{d}{d t}\right) \ell$ be the image representation of the controllable part of the behavioural system defined by $R\left(\frac{d}{d t}\right) w=0$. Hence, $R(\xi) M(\xi)=0$. It follows from (8) that

$$
M(-i \omega)^{T} \partial \Phi(i \omega) M(i \omega) \geq 0 \quad \forall \omega \in \mathbb{R} .
$$

Thus, given any $w \in \mathfrak{D}\left(\mathbb{R}, \mathbb{R}^{q}\right)$ such that $R\left(\frac{d}{d t}\right) w=0$, then we can write $w=M\left(\frac{d}{d t}\right) \ell$. Hence, using Parseval's Theorem,

$$
\begin{aligned}
& \int_{-\infty}^{\infty} Q_{\Phi}(w) d t \\
& =\int_{-\infty}^{\infty} \hat{\ell}(-i \omega)^{T} M(-i \omega)^{T} \partial \Phi(i \omega) M(i \omega) \hat{\ell}(i \omega) d \omega \\
& \geq 0
\end{aligned}
$$

where $\hat{\ell}(i \omega)$ is the Fourier Transform of $\ell(t)$. Thus, (7) holds.

To prove the converse part of the lemma, suppose that condition (8) does not hold. That is, there exists a $a \in$ $\mathbb{C}, m(\xi) \in \mathbb{R}^{q}[\xi]$ and $\omega_{0} \in \mathbb{R}$ such that $R(\xi) m(\xi)=0$ and

$$
a^{*} m\left(-i \omega_{0}\right)^{T} \partial \Phi\left(i \omega_{0}\right) m\left(i \omega_{0}\right) a<0 .
$$

Also, define a sequence of functions $\ell_{N}(t) \in \mathfrak{D}\left(\mathbb{R}, \mathbb{C}^{r}\right)$, $N=1,2, \ldots$, by

$$
\ell_{N}(t)= \begin{cases}e^{i \omega_{0} t} a & \text { for }|t| \leq \frac{2 \pi N}{\omega_{0}}, \\ \tilde{l}\left(t+\frac{2 \pi N}{\omega_{0}}\right) & \text { for } t<-\frac{2 \pi N}{\omega_{0}}, \\ \tilde{l}\left(t-\frac{2 \pi N}{\omega_{0}}\right) & \text { for } t>\frac{2 \pi N}{\omega_{0}} .\end{cases}
$$

Here $\tilde{l}(\cdot)$ is a function chosen independently of $N$ so that $l_{N}(t) \in \mathfrak{D}\left(\mathbb{R}, \mathbb{C}^{q}\right)$ for all $N$. This is possible because of the periodic nature of $\ell_{N}(t)$ for $|t| \leq$ $\frac{2 \pi N}{\omega_{0}}$. Also, define a corresponding sequence of functions $w_{N}(t) \in \mathfrak{D}\left(\mathbb{R}, \mathbb{C}^{r}\right), N=1,2, \ldots$, so that $w_{N}=$ $m\left(\frac{d}{d t}\right) \ell_{N}$. It follows from $R(\xi) m(\xi)=0$ that

$$
R\left(\frac{d}{d t}\right) w_{N}=0
$$

for all $N$. Also, we can write

$$
\begin{aligned}
& \int_{-\infty}^{\infty} Q_{\Phi}\left(w_{N}\right) d t \\
& =\frac{4 \pi N}{\omega_{0}} a^{*} m\left(-i \omega_{0}\right)^{T} \partial \Phi\left(i \omega_{0}\right) m\left(i \omega_{0}\right) a+E
\end{aligned}
$$

where $E$ is independent of $N$. Thus, it follows from (9) that for sufficiently large $N$,

$$
\int_{-\infty}^{\infty} Q_{\Phi}\left(w_{N}\right) d t<0 .
$$

A similar conclusion can be obtained using a real signal $\ell(t)$ by taking real and imaginary parts of $\ell_{N}$. Thus, condition (7) does not hold. This completes the proof of the lemma.

PROOF OF THEOREM 5. We consider two cases:

Case 1. There exists an $\omega \in \mathbb{R}$ such that $\partial \Phi_{1}(i \omega) \not \leq 0$. In this case, condition $(i i)$ of the theorem cannot hold so we prove $\Sigma_{\Phi_{1}} \leq \Sigma_{\Phi_{2}}$ if and only if condition $(i)$ of the theorem holds. Indeed, suppose that (5) holds. Hence

$$
\partial \Phi_{2}(i \omega)-\tau \partial \Phi_{1}(i \omega) \geq 0 \quad \forall \omega \in \mathbb{R} .
$$

Then it follows from Part 1. of Lemma 4 that

$$
\int_{-\infty}^{\infty}\left(Q_{\Phi_{2}}(w)-\tau Q_{\Phi_{1}}(w)\right) d t \geq 0 \quad \forall w \in \mathfrak{D}\left(\mathbb{R}, \mathbb{R}^{q}\right) .
$$

For any $w \in \mathfrak{B}_{\Phi_{1}}$, it follows that

$$
\int_{-\infty}^{\infty} Q_{\Phi_{1}}(w) d t \geq 0
$$

Hence, using (10), we conclude

$$
\begin{aligned}
\int_{-\infty}^{\infty} Q_{\Phi_{2}}(w) d t & \geq \tau \int_{-\infty}^{\infty} Q_{\Phi_{1}}(w) d t \\
& \geq 0 .
\end{aligned}
$$

Thus, $w \in \mathfrak{B}_{\Phi_{2}}$. However, since $w \in \mathfrak{B}_{\Phi_{1}}$ was arbitrary, we conclude that $\mathfrak{B}_{\Phi_{1}} \subset \mathfrak{B}_{\Phi_{2}}$. That is, $\Sigma_{\Phi_{1}} \leq$ $\Sigma_{\Phi_{2}}$.

We now suppose $\Sigma_{\Phi_{1}} \leq \Sigma_{\Phi_{2}}$. That is, $w \in \mathfrak{D}\left(\mathbb{R}, \mathbb{R}^{q}\right)$ such that

$$
\int_{-\infty}^{\infty} Q_{\Phi_{1}}(w) d t \geq 0
$$

implies

$$
\int_{-\infty}^{\infty} Q_{\Phi_{2}}(w) d t \geq 0
$$

Now define quadratic functionals $\mathscr{G}_{1}, \mathscr{G}_{2}$ on $\mathfrak{D}\left(\mathbb{R}, \mathbb{R}^{q}\right)$ as follows:

$$
\begin{aligned}
& \mathscr{G}_{1}(w):=\int_{-\infty}^{\infty} Q_{\Phi_{1}}(w) d t, \\
& \mathscr{G}_{2}(w):=\int_{-\infty}^{\infty} Q_{\Phi_{2}}(w) d t .
\end{aligned}
$$

It follows that $\mathscr{G}_{2}(w) \geq 0$ for all $w \in \mathfrak{D}\left(\mathbb{R}, \mathbb{R}^{q}\right)$ such that $\mathscr{G}_{1}(w) \geq 0$. Also, it follows from Lemma 6 that 
there exists a $\omega \in \mathbb{R}$ such that $\mathscr{G}_{1}(w)>0$. Hence, it follows from Lemma 3 that there exists a constant $\tau \geq$ 0 such that $\mathscr{G}_{2}(w)-\tau \mathscr{G}_{1}(w) \geq 0$ for all $w \in \mathfrak{D}\left(\mathbb{R}, \mathbb{R}^{q}\right)$. That is

$$
\int_{-\infty}^{\infty}\left(Q_{\Phi_{2}}(w)-\tau Q_{\Phi_{1}}(w)\right) d t \geq 0
$$

for all $w \in \mathfrak{D}\left(\mathbb{R}, \mathbb{R}^{q}\right)$. Thus, it follows from Part 2. of Lemma 4 that

$$
\partial \Phi_{2}(i \omega)-\tau \partial \Phi_{1}(i \omega) \geq 0 \quad \forall \omega \in \mathbb{R} .
$$

That is, condition (5) is satisfied. This completes the proof for case 1 .

Case 2. $\partial \Phi_{1}(i \omega) \leq 0$ for all $\omega \in \mathbb{R}$. In this case $\mathfrak{B}_{\Phi_{1}}$ is the controllable part of the behavioural system $\left\{w \in \mathfrak{D}\left(\mathbb{R}, \mathbb{R}^{q}\right): \partial \Phi_{1}\left(\frac{d}{d t}\right) w=0\right\}$. Hence, it follows from Lemma 7 that

$$
\mathfrak{B}_{\Phi_{1}} \subset \mathfrak{B}_{\Phi_{2}}
$$

if and only if

$$
m(-i \omega)^{T} \partial \Phi_{2}(i \omega) m(i \omega) \geq 0 \quad \forall \omega \in \mathbb{R}
$$

for all $m(\xi) \in \mathbb{R}^{q}[\xi]$ such that $\partial \Phi_{1}(\xi) m(\xi)=0$. That is, condition $(i i)$ holds. This completes the proof case 2.

\section{EXAMPLE}

In this section, we present an example which illustrates the above results: We will illustrate Theorem 5 by considering two differential inequality systems of the form defined in Definition 1. Our first system corresponds to the linear input-output system with transfer function:

$$
\frac{Y(s)}{U(s)}=\frac{1}{(1+s)^{2}}
$$

If we write

$$
w=\left[\begin{array}{l}
u \\
y
\end{array}\right],
$$

then this transfer function corresponds to the following behavioural constraint:

$$
R_{1}\left(\frac{d}{d t}\right) w=0
$$

where $R_{1}(s)=\left[\begin{array}{ll}1 & -(1+s)^{2}\end{array}\right]$. Then as in (2), we can define a corresponding differential inequality system $\Sigma_{\Phi_{1}}$ with

$$
\begin{aligned}
\Phi_{1}(\zeta, \eta) & =-R_{1}^{T}(\zeta) R_{1}(\eta) \\
& =\left[\begin{array}{cc}
-1 & (1+\eta)^{2} \\
(1+\zeta)^{2} & -(1+\eta)^{2}(1+\zeta)^{2}
\end{array}\right]
\end{aligned}
$$

To define our second differential system, consider a linear input-output system with transfer function

$$
\frac{Y(s)}{U(s)}=\frac{1-\delta_{1}}{s-\delta_{2}}
$$

where $\delta_{1}$ and $\delta_{2}$ are real uncertain parameters satisfying the bound $\delta_{1}^{2}+\delta_{2}^{2} \leq 1$. We can 'overbound' the behaviour corresponding to this collection of transfer functions by an differential inequality system of the form defined in Definition 1 as follows: Let

$$
w=\left[\begin{array}{l}
u \\
y
\end{array}\right] \text {. }
$$

Then the above transfer function implies the following behavioural constraint:

$$
\left[\begin{array}{ll}
1 & -\frac{d}{d t}
\end{array}\right] w=\left[\begin{array}{ll}
\delta_{1} & \delta_{2}
\end{array}\right]\left[\begin{array}{cc}
1 & 0 \\
0 & -1
\end{array}\right] w .
$$

Hence,

$$
\begin{aligned}
& \int_{-\infty}^{\infty}\left(R_{2}\left(\frac{d}{d t}\right) w\right)^{T} R_{2}\left(\frac{d}{d t}\right) w d t \\
& =\int_{-\infty}^{\infty} w^{T} T_{2}^{T}\left(\delta_{1}^{2}+\delta_{2}^{2}\right) T_{2} w d t \\
& \leq \int_{-\infty}^{\infty} w^{T} T_{2}^{T} T_{2} w d t
\end{aligned}
$$

where

$$
R_{2}(s)=\left[\begin{array}{ll}
1 & -s
\end{array}\right] ; \quad T_{2}=\left[\begin{array}{cc}
1 & 0 \\
0 & -1
\end{array}\right]
$$

Then as in Section 2, we can define a corresponding differential inequality system $\Sigma_{\Phi_{2}}$ with

$$
\Phi_{2}(\zeta, \eta)=T_{2}^{T} T_{2}-R_{2}^{T}(\zeta) R_{2}(\eta)=\left[\begin{array}{cc}
0 & \eta \\
\zeta & 1-\eta \zeta
\end{array}\right]
$$

We now use Theorem 5 to show that $\Sigma_{\Phi_{1}} \leq \Sigma_{\Phi_{2}}$. Indeed, we calculate

$$
\partial \Phi_{1}(i \omega)=\left[\begin{array}{cc}
-1 & 1+2 i \omega-\omega^{2} \\
1-2 i \omega-\omega^{2} & -1-2 \omega^{2}-\omega^{4}
\end{array}\right]
$$

and

$$
\partial \Phi_{2}(i \omega)=\left[\begin{array}{cc}
0 & i \omega \\
-i \omega & 1-\omega^{2}
\end{array}\right]
$$

Thus,

$$
\begin{aligned}
& \partial \Phi_{2}(i \omega)-\tau \partial \Phi_{1}(i \omega) \\
& =\left[\begin{array}{cc}
1 & -1-i \omega+\omega^{2} \\
-1+i \omega+\omega^{2} & 2+\omega^{2}+\omega^{4}
\end{array}\right] \geq 0
\end{aligned}
$$

for $\tau=1$. Hence, using Theorem 5, we can conclude that $\Sigma_{\Phi_{1}} \leq \Sigma_{\Phi_{2}}$.

\section{REFERENCES}

Beck, C. (1994). Minimality for uncertain systems with IQCs. In: Proceedings of the 33rd IEEE Conference on Decision and Control. Lake Buena Vista, Florida. pp. 3068-3073. 
Beck, C. L. and J. C. Doyle (1999). A necessary and sufficient minimality condition for uncertain systems. IEEE Transactions on Automatic Control 44(10), 1802-1813.

Beck, C. L. and R. D'Andrea (1997). Minimality, controllability and observability for uncertain systems. In: Proceedings of the 1997 American Control Conference. pp. 3130-3135.

Beck, C. L., J. C. Doyle and K. Glover (1996). Model reduction of multidimensional and uncertain systems. IEEE Transactions on Automatic Control.

Boyd, S., L. El Ghaoui, E. Feron and V. Balakrishnan (1994). Linear Matrix Inequalities in System and Control Theory. SIAM. Philadelphia, Pennsylvania.

D'Andrea, R. and F. Paganini (1993). Interconnection of uncertain behavioral systems for robust control. In: Proceedings of the 32rd Conference on Decisiona and Control. San Antonio, Texas. pp. 3642-3647.

D'Andrea, R. and F. Paganini (1994). Controller synthesis for implicitly defined uncertain systems. In: Proceedings of the 33rd Conference on Decisiona and Control. Lake Buena Vista, FL. pp. 3679-3684.

D’Andrea, R., F. Paganini and J. C. Doyle (1993). Uncertain behavior. In: Proceedings of the 32rd Conference on Decisiona and Control. San Antonio, Texas. pp. 3891-3896.

Megretski, A. and A. Rantzer (1997). System analysis via integral quadratic constraints. IEEE Transactions on Automatic Control 42(6), 819-830.

Paganini, F. and J. C. Doyle (1994). Analysis of implicitly defined systems. In: Proceedings of the 33rd Conference on Decisiona and Control. Lake Buena Vista, FL. pp. 3673-3678.

Petersen, I. R., V. Ugrinovski and A. V. Savkin (2000). Robust Control Design using $H^{\infty}$ Methods. Springer-Verlag London.

Polderman, J. W. and J. C. Willems (1998). Introduction to Mathematical Systems Theory: A Behavioral Approach. Springer. New York.

Willems, J. C. and H. L. Trentelman (1998). On quadratic differential forms. SIAM Journal on Control and Optimization 36(5), 1703-1749.

Yakubovich, V. A. (1973). Minimization of quadratic functionals under the quadratic constraints and the necessity of a frequency condition in the quadratic criterion for absolute stability of nonlinear control systems. Soviet Mathematics Doklady 14, 593-597. 